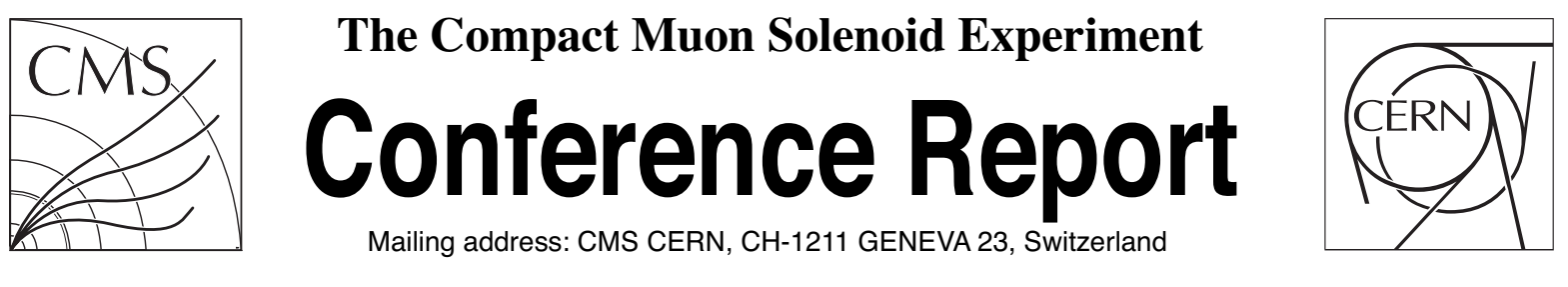

04 February 2019 (v5, 16 July 2019)

\title{
The Upgrade of the CMS Inner Tracker for HL-LHC
}

Malte Backhaus for the CMS collaboration

\begin{abstract}
During the Long Shutdown (LS) from 2024 to 2026 the Large Hadron Collider (LHC) will be upgraded in order to provide a ten-fold increase of the data rate to the experiments. This will enhance the sensitivity of the experiments to possible unexplored rare processes and to allow for precision measurements of known processes, in particular of the properties of the Higgs Boson which was discovered in 2012. This accelerator upgrade project is called High-Luminosity LHC (HL-LHC). The entire silicon tracker of the Compact Muon Solenoid (CMS) experiment will be replaced for the HL-LHC with a new detector featuring increased radiation hardness during the collection of above $3000 \mathrm{fb}^{-1}$ of integrated luminosity and capability to handle the higher data rate at an instantaneous luminosity of $10^{35} \mathrm{~cm}^{-2} \mathrm{~s}^{-1}$ and longer trigger latency of $12.5 \mu \mathrm{s}$. The Inner Tracker will be exposed to radiation levels up to a non ionizing energy loss equivalent to $2.3 \times 10^{16} \mathrm{n}_{\mathrm{eq}} / \mathrm{cm}^{2}$ and an accumulated ionizing dose of $1.2 \mathrm{Grad}$, requiring advanced technologies and novel solutions among others for the readout and powering electronics. The new detector will also feature extended pseudo-rapidity $\eta$ coverage with a tracking volume up to $\eta=4$. The High-Luminosity upgrade of the CMS silicon pixel detector is reviewed in this paper. The novel features of the detector layout and electronics system are discussed, and the expected performance is summarized.
\end{abstract}

Presented at PIXEL2018 International Workshop on Semiconductor Pixel Detectors for Particles and Imaging 2018 


\title{
The Upgrade of the CMS Inner Tracker for HL-LHC
}

\author{
M. Backhaus on behalf of CMS collaboration \\ ETH Zürich, Rämistrasse 101, 8092 Zürich, Switzerland \\ E-mail: backhaus@cern.ch
}

\begin{abstract}
During the Long Shutdown (LS) from 2024 to 2026 the Large Hadron Collider (LHC) will be upgraded in order to provide a ten-fold increase of the data rate to the experiments. This will enhance the sensitivity of the experiments to possible unexplored rare processes and to allow for precision measurements of known processes, in particular of the properties of the Higgs Boson which was discovered in 2012. This accelerator upgrade project is called High-Luminosity LHC (HL-LHC). The entire silicon tracker of the Compact Muon Solenoid (CMS) experiment will be replaced for the HL-LHC with a new detector featuring increased radiation hardness during the collection of above $3000 \mathrm{fb}^{-1}$ of integrated luminosity and capability to handle the higher data rate at an instantaneous luminosity of $10^{35} \mathrm{~cm}^{-2} \mathrm{~s}^{-1}$ and longer trigger latency of $12.5 \mu \mathrm{s}$. The Inner Tracker will be exposed to radiation levels up to a non ionizing energy loss equivalent to $2.3 \times 10^{16} \mathrm{n}_{\mathrm{eq}} / \mathrm{cm}^{2}$ and an accumulated ionizing dose of $1.2 \mathrm{Grad}$, requiring advanced technologies and novel solutions among others for the readout and powering electronics. The new detector will also feature extended pseudo-rapidity $\eta$ coverage with a tracking volume up to $\eta=4$. The High-Luminosity upgrade of the CMS silicon pixel detector is reviewed in this paper. The novel features of the detector layout and electronics system are discussed, and the expected performance is summarized.
\end{abstract}

KeYwords: Solid state detectors, Large detector systems for particle and astroparticle physics, Particle tracking detectors (Solid-state detectors), Detector design and construction technologies and materials 


\section{Contents}

1 Experimental conditions for CMS at HL-LHC 1

2 The CMS Tracker for HL-LHC 2

$3 \quad$ Inner Tracker sensor design and testing 3

4 Inner Tracker readout chip 4

5 Serial powering for the CMS Inner Tracker 6

$\begin{array}{llr}6 & \text { Inner Tracker module developments } & 8\end{array}$

7 Mechanical support structure and material budget of the Inner Tracker 9

8 Expected tracking performance $\quad 10$

9 Conclusions $\quad 10$

\section{Experimental conditions for CMS at HL-LHC}

The sensitivity for discovery of very rare events as well as high precision measurements of known processes increase significantly with larger data sets recorded by the experiments. While a high instantaneous luminosity of the accelerator enables a high data recording rate, it poses significant experimental challenges to the detector systems. The instantaneous luminosity of the Large Hadron Collider (LHC) [1] at CERN ${ }^{1}$ is gradually increased during its operational time. The original design value of the LHC is $10^{34} \mathrm{~cm}^{-2} \mathrm{~s}^{-1}$ [1]. The design luminosity was surpassed by more than a factor of two in November 2017 with a maximum luminosity of $2.066 \times 10^{34} \mathrm{~cm}^{-2} \mathrm{~s}^{-1}$ [2] recorded by the Compact Muon Solenoid (CMS) experiment. During the Long Shutdown of 2024 to 2026, the LHC will prepare for its final luminosity increase. After this major upgrade the instantaneous luminosity will be about $10^{35} \mathrm{~cm}^{-2} \mathrm{~s}^{-1}$ [3] during the subsequent High Luminosity LHC (HL-LHC) [3] operation period. Data acquisition at this high rate will enable to collect up to $4000 \mathrm{fb}^{-1}$ during ten years of operation. The operational conditions will be challenging, especially for the tracking systems. At this luminosity about 10000 charged particles arise every $25 \mathrm{~ns}$ from up to 200 simultaneous collisions per bunch crossing.

\footnotetext{
${ }^{1}$ European Organization for Nuclear Research
} 


\section{The CMS Tracker for HL-LHC}

A completely new all silicon Tracker [4] is being developed for the CMS experiment at HLLHC. The tracker system will be operated in a $3.8 \mathrm{~T}$ magnetic field and will contribute to the trigger information using the following concept: complex silicon strip-strip and strip-pixel modules provide immediate transverse momentum information extracted from the track curvature in the magnetic field. Depending on the curvature of its track a particle produces hits in a two subsequently mounted sensors. The distance of the hits allows the discrimination of high-momentum (low curvature, small distance) and lower momentum (larger curvature, larger distance) in the on-detector hardware, see figure 1. Only hits surpassing a transverse momentum threshold are used as so-called stubs for

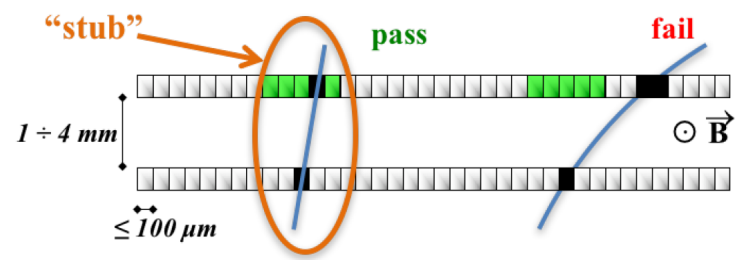

Figure 1. Illustration of the $p_{T}$-module concept. Correlation of signals in closely-spaced sensors enables rejection of low- $p_{T}$ particles; the channels shown in green represent the selection window to define an accepted stub. Adapted from [4].

extremely fast track reconstruction. These tracks with high transverse momentum are then included in the trigger decision to readout the full CMS detector. The readout electronics of all other detector systems need to be operated with the new trigger latency which is increased from $3.75 \mu \mathrm{s}$ to $12.5 \mu \mathrm{s}$ and a trigger rate up to $750 \mathrm{kHz}$ [4]. Especially for the Inner Tracker, consisting of hybrid silicon pixel modules, this long latency is challenging due to the extreme particle hit rate of up to $3.5 \mathrm{GHz} / \mathrm{cm}^{2}$ [4]. Until the end of operation the expected accumulated Total Ionizing Dose (TID) is up to $1.2 \mathrm{Grad}$ and the fluence is up to $2.3 \times 10^{16} \mathrm{n}_{\mathrm{eq}} / \mathrm{cm}^{2}$ [4].

The layout of the CMS Tracker consists of a barrel - horizontally flat part - closed by two end-caps. The barrel covers the volume from $z=0 \mathrm{~mm}$ to $z=1200 \mathrm{~mm}$ for the Outer Tracker and $z=0 \mathrm{~mm}$ to $z=220 \mathrm{~mm}$ for the Inner Tracker of the CMS Tracker for HL-LHC consists of ten silicon layers [4] as shown in figure 2. Six of the layers provide $\mathrm{p}_{T}$ information in addition to space-points. Layers eight to ten (red in the figure) consist of strip-strip modules, and layers five to seven (blue in the figure) of strip-pixel modules. As illustrated in the figure the barrel section of the strip-pixel modules is gradually inclined in the range of pseudo-rapidity $\eta$ between 0.6 and 2.2. The Inner Tracker barrel (TBPX) holds layer one to four and consists of hybrid silicon pixel modules. The barrel part of the Tracker is complemented at larger $\eta$ by endcap discs. The Outer Tracker endcap holds five discs between $z=1200 \mathrm{~mm}$ and $z=2700 \mathrm{~mm}$, and the Inner Tracker endcap holds eight small discs in the forward section (TFPX) from $z=250 \mathrm{~mm}$ to $z=1600 \mathrm{~mm}$ with four rings of modules each, and four large discs in the high $\eta$ extension section (TEPX) of $z=1600 \mathrm{~mm}$ to $z=2600 \mathrm{~mm}$ with five rings of modules. This layout together with the novel features of the Inner Tracker described in section 3 to 7 provides a highly efficient tracking volume extending until $\eta=4$ (more details in section 8).

The outer radius of the Inner Tracker (IT) volume increases at $z \simeq 1600 \mathrm{~mm}$ (see figure 2). The 


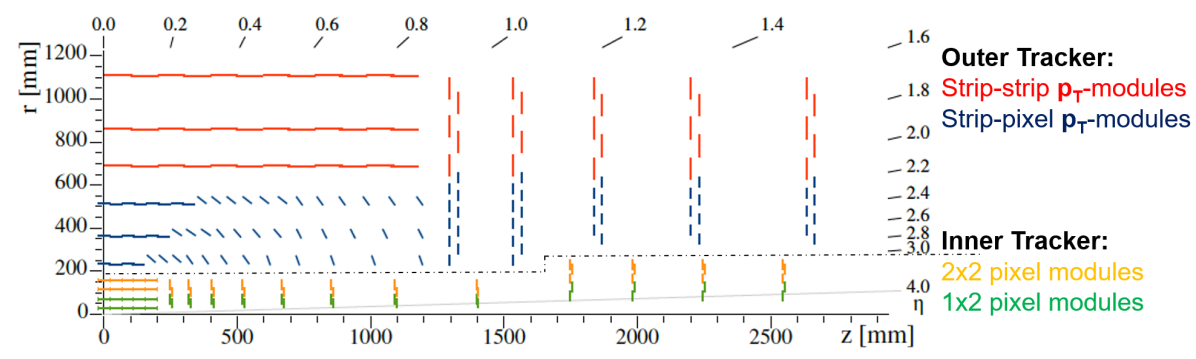

Figure 2. Layout of the CMS Tracker for HL-LHC (adapted from [4]). One quarter of the Tracker is shown. The origin of the figure corresponds to the center of the region in which the bunches collide. The beam pipe is aligned with the $z$-axis.

installation of the new detector inside the current CMS is required from both ends. The structure is divided in eight packages, which are subsequently inserted. The TBPX and TFPX build a joint structure installed in four shells. Afterwards the TEPX will be installed in four quarters. Figure 3 shows one complete quarter of the pixel detector and depicts the different sections. This paper concentrates in the following on the Inner Tracker, and concludes with a presentation of the expected performance of the full silicon Tracker.

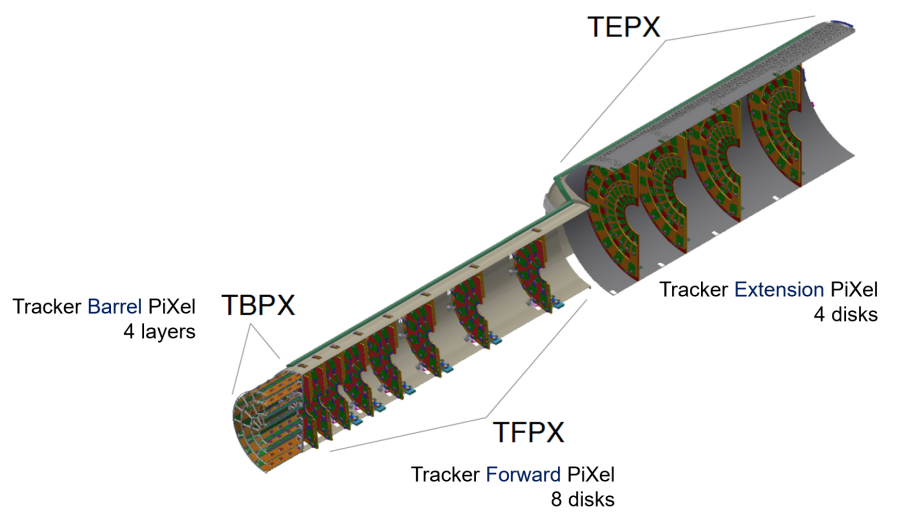

Figure 3. Sketch of one quarter of the CMS Tracker for HL-LHC (adapted from [4]).

\section{Inner Tracker sensor design and testing}

The CMS pixel sensor $\mathrm{R} \& \mathrm{D}^{2}$ investigates different sensor concepts in an extensive irradiation and characterization campaign to mimic the conditions of HL-LHC. During 2018 and 2019 a total of 88 sensors bump-bonded to prototype readout chips have been irradiated on dedicated lightweight carrier boards and characterized in test beams (see figure 4).

The sensors under investigation are 3D [5] and n-in-p planar sensors. The variety of 3D sensors under investigation is described in [6] in the same issue. The planar sensor R\&D concentrates on sensors with an active thickness between $100 \mu \mathrm{m}$ and $150 \mu \mathrm{m}$, which results in an expected signal over threshold larger than three at a fluence of $8 \times 10^{15} \mathrm{n}_{\mathrm{eq}} / \mathrm{cm}^{2}$ [7]. Due to the extreme track

${ }^{2}$ Research and Development 


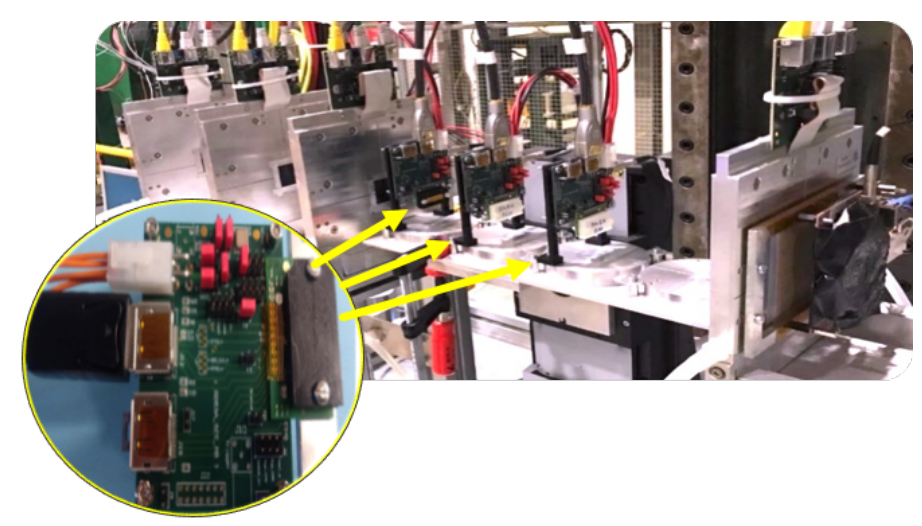

Figure 4. Photo of the test beam setup and close-up of the dedicated lightweight carrier board for irradiation and test of sensor-chip assemblies [7].
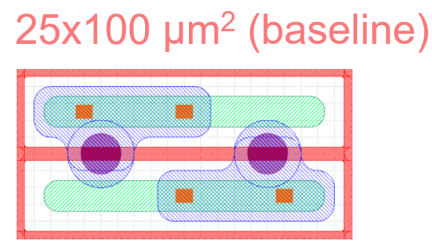

$50 \times 50 \mu m^{2}$

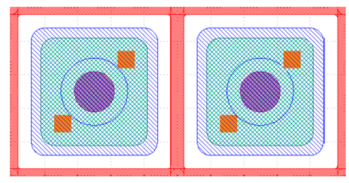

Figure 5. Illustration of sensor cell designs of dimensions $25 \times 100 \mu \mathrm{m}^{2}$ (left) and $50 \times 50 \mu \mathrm{m}^{2}$ (right) for connection to the readout-chip with $50 \mu \mathrm{m}$ bump pitch in both directions [4]. The p-stop inter-pixel isolation areas are shown in orange, the $n$-doped implants of the readout electrodes in green, the metal layer in light purple, the bump pad opening in purple, and the orange squares indicate the connection of the metal to the readout electrodes.

density a pixel cell size reduction by a factor of six with respect to the current CMS pixel detector is planned. This helps to limit the occupancy below the requirement of the permille level [4] and to reduce inefficiencies due to successive hits in the same pixel (see also [8]). Illustrations of the pixel cell designs under investigation are shown in figure 5.

Several bias structures ranging from independent bias per pixel over a common bias structure for four pixels to no bias structure are prototyped. Preliminary results of test beam campaigns show a very good global efficiency of $99.8 \%$ for the common punch-through design (see figure 6 left) [9]. A drop of the efficiency is observed at the position of the punch-through bias dot if the bias grid is grounded [9] (see figure 6 right). This result motivates the use of a floating bias structure after flip-chip. The result is confirmed by several further measurements such as a measurement of the charge sharing between the pixels [7].

\section{Inner Tracker readout chip}

The pixel readout chip is developed by the CERN RD53 collaboration [10], which develops all needed IP blocks and designs the readout chips for upgrades of the ATLAS and the CMS pixel detectors. The chips are designed in a $65 \mathrm{~nm}$ technology. This small size achieves the logic density 


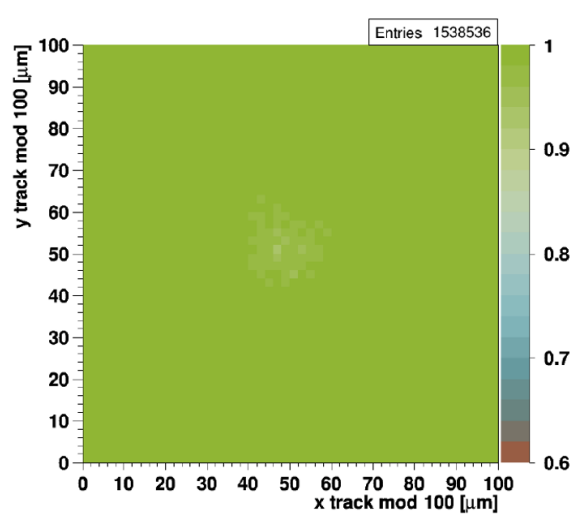

Biad Grid Floating $99.8 \%$

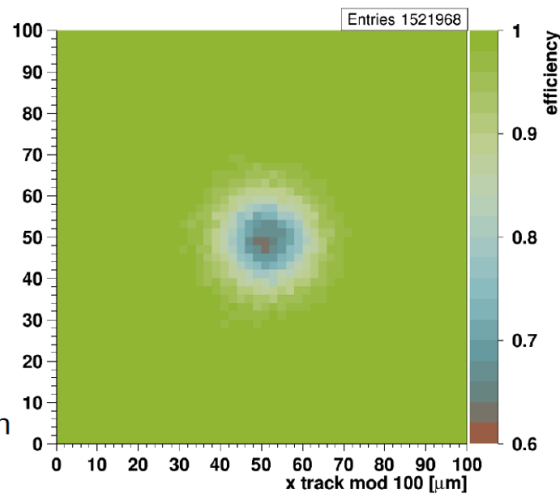

Bias Grid Grounded $98.4 \%$

Figure 6. Measurement of the efficiency as a function of position for different sensor cell bias structure configurations. The four sensor cells have a common punch-through bias dot as illustrated in the center. The left graph corresponds to a bias grid that was left floating during the measurement, while the bias grid was fixed to the readout-chip ground potential during the measurement presented in the graph on the right. Adapted from [9].

needed for efficient buffering of the high hit occupancy during the long trigger latency. The layout of the analog logic is done manually and the analog circuitry of four pixels are placed in one analog island. All digital logic is synthesized and forms a digital sea in which the analog islands are placed [10]. Figure 7 illustrates this design concept.
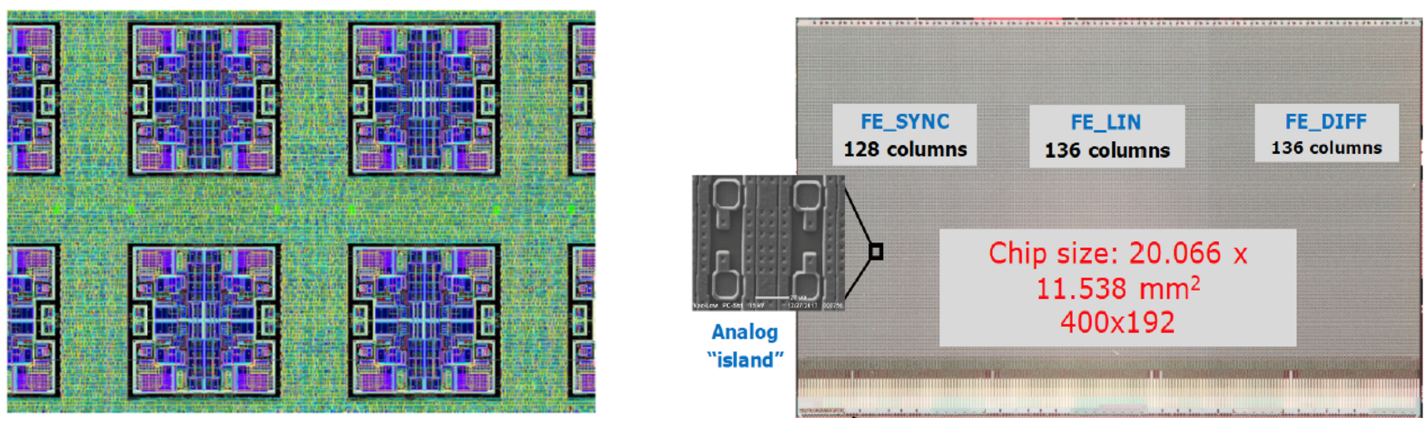

Figure 7. Left: illustration of the placement of analog islands (blue areas) containing the analog logic of four pixels in a digital sea (green area). The digital sea contains all synthesized digital logic such as latency counters and hit buffers [11]. Right: photo of the chip and indication of the three matrices with different analog circuitries.

The first prototype chip $R D 53 A$ contains an active matrix of $400 \times 192$ pixels. This is about half the size of the final readout chip, but timing and voltage drop requirements are scaled to final chip size. Three different analog circuitries are prototyped in three sub-matrices, see figure 7 on the right. The threshold in terms of signal charge at the input for detector operation is expected to be below $1000 \mathrm{e}$. All three sub-matrices can be operated with this upper threshold limit [12]. Figure 8 shows a pixel-to-pixel threshold distribution for the linear Front-End prototype matrix as well as the noise distribution. A good threshold uniformity with a mean threshold below $1000 \mathrm{e}$ is achieved. The mean noise is $67 \mathrm{e}$ with a pixel-to-pixel variation of only $4 \mathrm{e}$. These values are 
calculated using the $\mathrm{DAC}^{3}$ to charge calibration as extracted from the chip simulation.
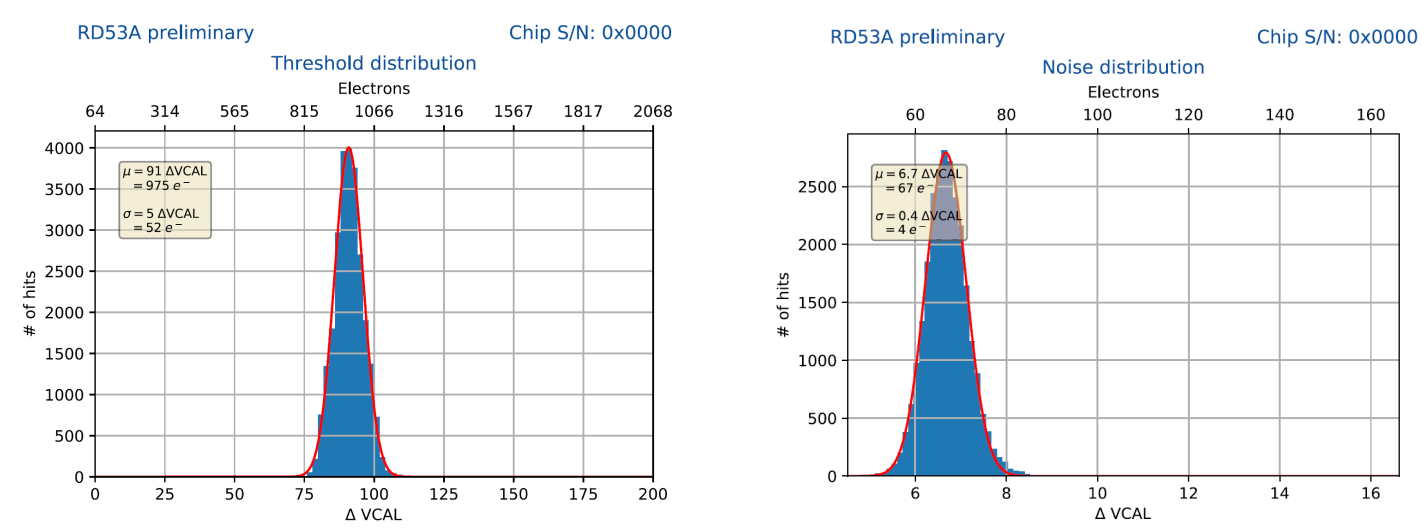

Figure 8. Left: pixel-to-pixel threshold distribution (left) and noise distribution (right) of the Linear Front-End sub-matrix [12].

The required TID tolerance is achieved using dedicated transistor models during synthesis that have been parameterized based on results from intensive irradiation campaigns [13, 14]. Figure 9 shows the result of one of the measurements done to test the operation of the chip against TID. The mean noise of the sub-matrix with the differential amplifier chain is measured for different operation modes of the chip, and the pixel-to-pixel distribution width is indicated with error bars. The chip was operational until the highest measured TID of $1 \mathrm{Grad}$. In all three modes the mean noise decreased by about $6 \%$ within the first $5 \mathrm{Mrad}$. For the range of $5 \mathrm{Mrad}$ to about $300 \mathrm{Mrad}$ the noise is constant. Above $300 \mathrm{Mrad}$ the noise decreases again by another $6 \%$. The width of the noise distribution is constant in the order of $10 \mathrm{e}$. The calibration for conversion from DAC to charge has not been calibrated during the result and a shift of the calibration instead of the noise can not be excluded at this point. Very similar results are obtained from all three sub-matrices. The chip is fully functional and meets the specifications also after $500 \mathrm{Mrad}$. The results indicate additionally that the operation up to a TID of $1 \mathrm{Grad}$ under controlled conditions (low temperature during the full life time) could be achieved.

Another important specification is the efficient hit buffering and processing in the high hit rate environment expected in the HL-LHC detectors. Dedicated measurements are performed and the procedure and results are reported in the same issue [8].

\section{Serial powering for the CMS Inner Tracker}

A conventional detector powering scheme supplies all chips in parallel with a constant voltage. The current consumption of the chips results in a voltage drop along the long supply cables. Fast current consumption variations therefore result in potentially lethal voltage transients at the inputs of the chips. The amplitude of the tolerable voltage transients in combination with the possible current variations defines the needed mass of the cables.

The CMS IT for HL-LHC will consist of 13488 chips [4] with a maximum supply current in the order of $2 \mathrm{~A}$ per chip at an input voltage of about $1.2 \mathrm{~V}$. The total power consumption of the

${ }^{3}$ Digital to Analog Converter 

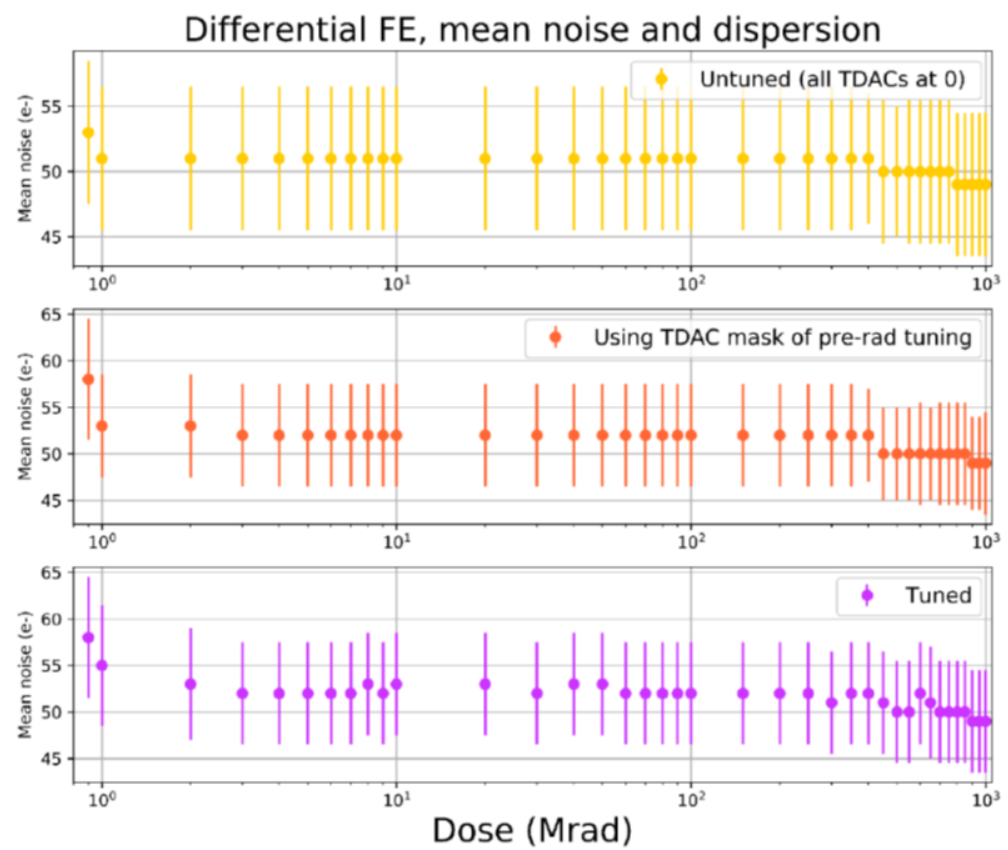

Figure 9. Measurement of the noise as a function of the TID. The mean noise (data points) and the width if the pixel-to-pixel distribution (error bars) of the differential Front-End matrix are shown for different operation modes.

detector is therefore above $30 \mathrm{~kW}$, which would require above six tons of cables, and still more than $2 / 3$ of the power delivered to the detector would be lost in the cables. A possible solution is to develop a system with a constant current consumption. This minimizes the transients by design and therefore the cable mass does not depend on the supply current and tolerable voltage transients. The needed supply current can be further reduced when the supply current delivered to a chip is re-used. For this the modules are connected in serial powering chains [15] of up to eleven modules [4]. A controlled splitting of the supply current between the chips on the module (connected in parallel) is needed and achieved by dedicated Shunt-LDO regulator blocks [16] implemented in the readout chip (see also [8] in the same issue). The ATLAS and CMS pixel detectors for HL-LHC are the first large scale application of serial powering in a detector system.

The geometrically most challenging section to implement serial powering chains of modules in the CMS IT is the TBPX section. Here the supply current must be routed from one end of the module to the other end and then the return current must be routed backwards along the chain. A module design with a power-pigtail is proposed (see also section 6), which plugs into the previous module. Figure 10 illustrates the connection of modules to a chain in TBPX on the left and shows a photo of the first chain of two prototype modules as described in section 6 . Intense characterization and validation measurements are being performed. These include the measurement of the impact 

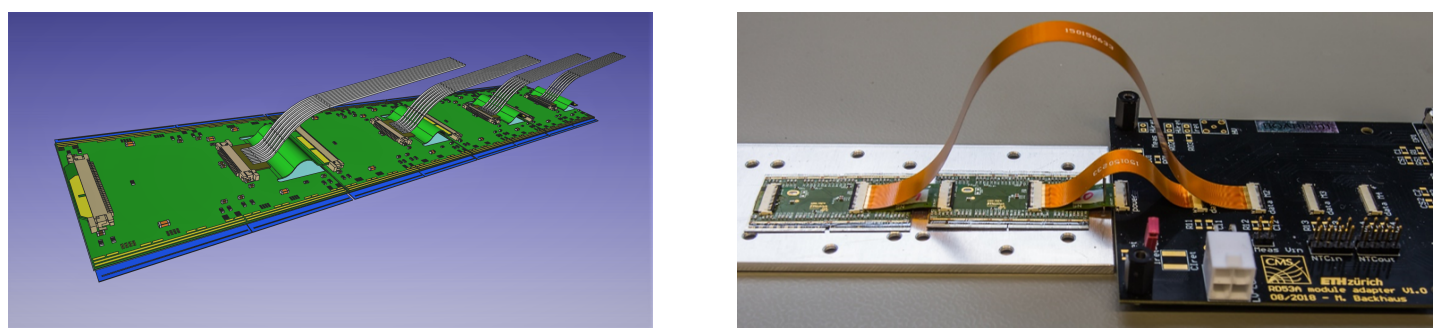

Figure 10. Left: illustration of the connection of four modules into a serial powering chain in the TBPX section. The chain is terminated on the left-most module using a dedicated flex-print. While power is routed from module to module, one independent cable serves as data link. Right: photo of the first prototype chain of two $2 \times 2$ RD53A modules (see section 6) connected to the test system.

of the powering scheme on chip characteristics as well as on detector-wide aspects. Details are reported in [8] in the same issue.

\section{Inner Tracker module developments}

The hybrid pixel modules will consist of a single silicon sensor which is bump-bonded to multiple readout chips. Depending on the position in the detector, a module has either two or four chips (see also section 1). The chips are placed in a $1 \times 2$ (one row, two columns) or in a $2 \times 2$ (two rows, two columns) arrangement. A printed flexible circuit, called High Density Interconnect (HDI), is glued on top of the sensor. The electrical connection of the readout chips and of the sensor bias voltage to the HDI is done with wire bonds. A first prototype of the HDI has been designed and produced in a small feature size flex technology. The flex is designed with three copper layers with a thickness below $10 \mu \mathrm{m}$ each. This allows for precise etching needed for the low feature size, which in turn allows a wire bond pattern without angles and different wire lengths. The HDI is compatible with serial connection to chains as presented in section 5 and can operate $2 \times 2$ RD53A chips. The routing of the supply current and of the return current with the thin copper layers is one of the major challenges. Figure 11 (left) shows a photo of the HDI. Detailed simulations of the supply current routing are used to optimize the power consumption of the HDI. The first version has a power consumption of $200 \mathrm{~mW}$ at a supply current of $5 \mathrm{~A}$ for four RD53A chips in the top layer (see figure 11 right), which is the most critical layer. This value is already within the expected power density per layer of the final HDI. A large fraction of the power consumption of the first prototype is concentrated in few localized areas. The design is currently being improved further to minimize the power consumption.

The HDI design has been verified before component loading using thermal images for the supply currents of the readout chips of up to $8 \mathrm{~A}$ and the sensor bias lines have been tested for leakage and sparking for voltages up to $1 \mathrm{kV}$. First operational prototype modules have been built. No sensor with a design for four RD53A chips was available at the time, and thus the sensor is replaced by a precisely cut piece of silicon, which is glued on top of the readout chips. With these digital modules the HDI design can be verified and the chip performance when connected to the HDI can be compared to the performance when loaded on massive test PCBs. Figure 12 (left) shows a photo of the first digital module. Intensive characterization is ongoing. To date no performance 

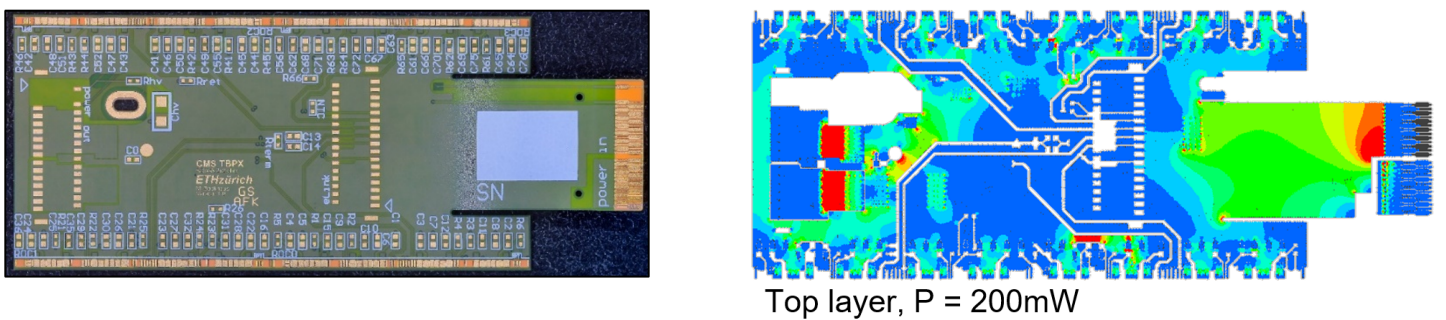

Figure 11. Left: photo of the HDI prototype fo $2 \times 2$ RD53A chips before population with components. Right: a simulated power density map of the most critical layer.

difference of the chips on HDIs with respect to chips on massive PCBs has been observed (see figure 12 right).
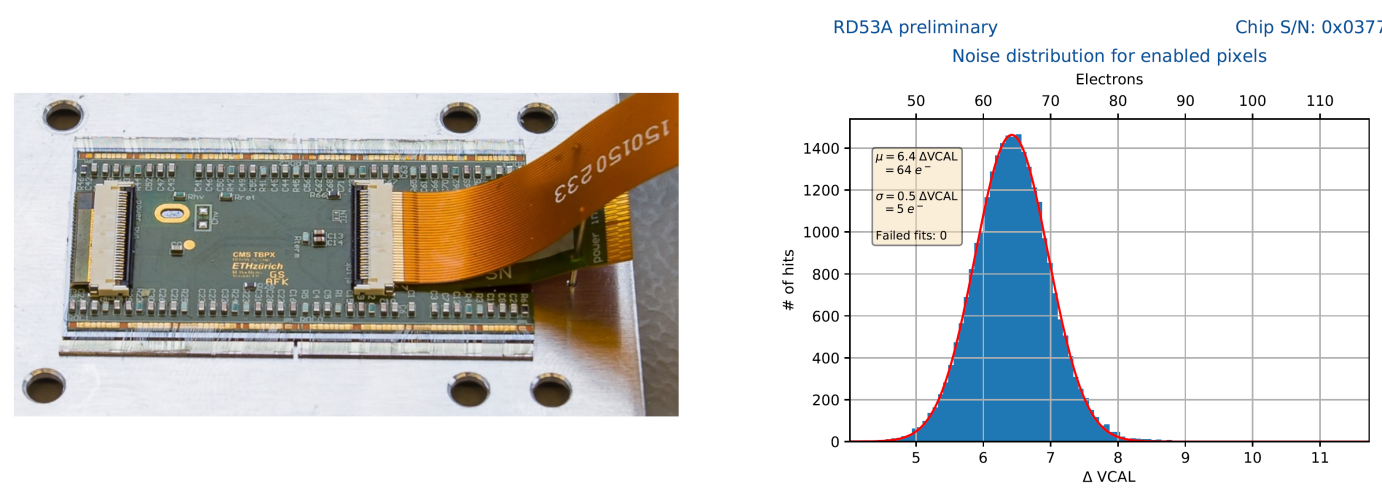

Figure 12. Left: photo of the first operational module build with four RD53A chips and the HDI prototype. The flat cable serves as data link to the test system. Right: pixel-to-pixel noise distribution of the linear Front-End of one of the RD53A chips on the digital module.

\section{Mechanical support structure and material budget of the Inner Tracker}

The mechanical support structure is subject to constant improvement. All three Inner Tracker sections are cooled using $\mathrm{CO}_{2}$-bi-phase cooling with a planned coolant temperature of $-33^{\circ} \mathrm{C}$ circulating in stainless steel pipes. For the TBPX a lightweight carbon ladder structure similar to the Phase 1 pixel detector [17] is being designed. Half-disc structures consisting of carbon foam and carbon laminates are investigated for the TFPX and TBPX sections. A preliminary material budget calculation has been performed as a function of the pseudo-rapidity. Figure 13 compares the material budget of the complete CMS Tracker for HL-LHC with the material budget of the current CMS Tracker. A reduction of up to $50 \%$ in material is achieved in the forward part, which is mainly due to improvements in the Outer Tracker. The Inner Tracker tracking volume is extended due to the TEPX until $\eta=4$, which is reflected in the additional material in the region with $\eta>2.5$. 

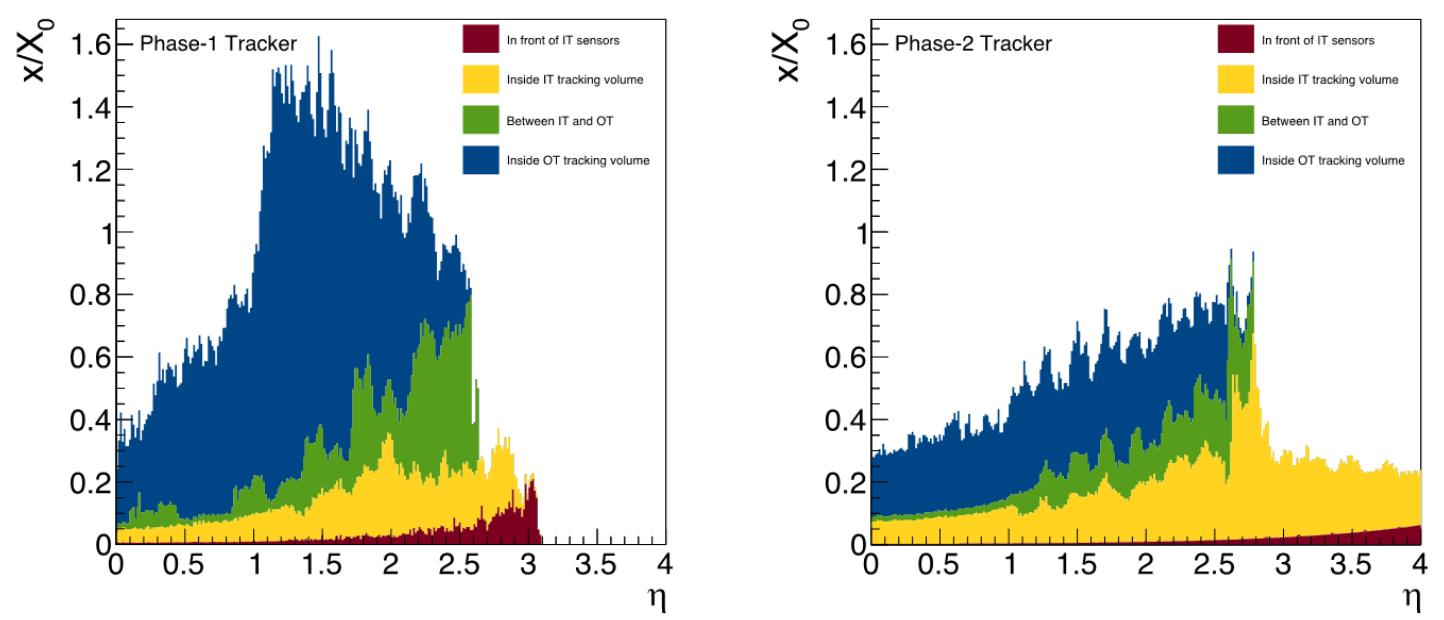

Figure 13. Comparison of the estimated material budget of the CMS Tracker as a function of pseudorapidity $\eta$. The material budget of the current CMS Tracker is shown on the left, and the estimated material budget of the Tracker for HL-LHC is shown on the right [4]. Material in front of the IT sensors is shown in red, material inside the IT tracking volume in yellow, material between the IT and the OT tracking volumes in green, and material in the OT tracking volume is shown in blue.

\section{Expected tracking performance}

Detailed tracking and physics performance simulations are carried out to validate the detector design [4]. Simulations of two of the most important performance characteristics (which are driven by the Inner Tracker) to achieve the physics performance needed to exploit the potential of the HL-LHC are shown in figure 14. The impact parameter resolution of muons with a transverse momentum of $10 \mathrm{GeV}$ of the CMS Tracker during HL-LHC operation (Phase 2) is compared to the impact parameter resolution of the CMS Tracker during LHC operation (Phase 1). Inside the full tracking volume of the Phase 1 Tracker $(\eta<2.5)$ the impact parameter resolution is reduced by a factor of about 2 (from about $150 \mu \mathrm{m}$ to about $80 \mu \mathrm{m}$ at $\eta=0$ ). Additionally, the extended tracking volume at $2.5<\eta<4$ with an impact parameter resolution below $200 \mu \mathrm{m}$ is visible. The simulated tracking efficiency for tracks with transverse momentum above $900 \mathrm{MeV}$ originating from top quark pairs is shown on the right side of figure 14 for the future HL-LHC operation. No significant difference is visible for scenarios with different amounts of simultaneous collisions per bunch-crossing (pile-up). The tracking efficiency is above $85 \%$ for nearly the full range of $0<\eta<4$ for both scenarios. This proves the robustness of the detector design in a high pile-up environment for the maximum expected luminosity resulting in 200 simultaneous collisions per bunch crossing.

\section{Conclusions}

The luminosity of the LHC will be increased in order to enhance the sensitivity to rare processes and increase the precision of measurements. The HL-LHC run period will start in 2026. A large number of technical challenges needs to be overcome in the design of the Inner Tracker for the CMS experiment for the HL-LHC. The high luminosity results in a high track density and extremely harsh 

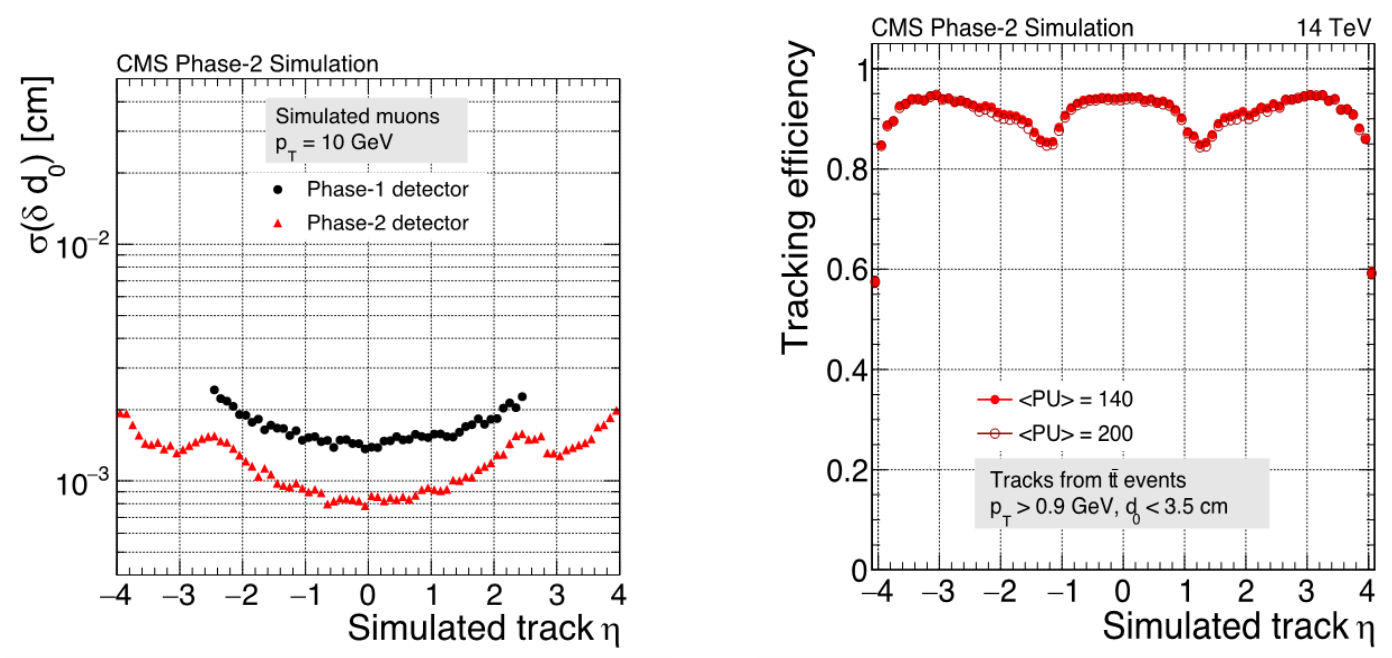

Figure 14. Left: Simulation of the impact parameter resolution for muon tracks with a transverse momentum above $10 \mathrm{GeV}$. The impact parameter resolution is shown as a function of $\eta$ for the Phase- 1 and the Phase- 2 detector [4]. Right: Simulation of the track finding efficiency as a function of eta for two different numbers of simultaneous collisions per bunch crossing [4].

radiation environment, which together with the long operation time requires unequaled radiation tolerance of the sensors and of the readout electronics.

Several novel techniques are exploited to address the challenges as well as to achieve a lightweight and efficient power distribution inside the detector. The presented results indicate the progress made in the development and characterization of these novel features, as well as advances towards the construction of realistic prototypes and system demonstrators. Most recent results of first prototype modules have been reported and demonstrate no performance degradation when the chips are operated on realistic pixel modules.

The design of all components as well as the layout is subject to steady improvements. Simulations of the tracking performance have been done using the particle-detector interaction properties with the foreseen material of the future tracker, and a model of the electrical performance of the modules. The results indicate a robust tracking performance in the challenging environment inside the CMS experiment during HL-LHC.

\section{References}

[1] O. Brüning, et al., LHC Design Report, CERN Yellow Reports: Monographs. CERN, Geneva, 2004.

[2] The CMS Collaboration, CMS Public Results: https://twiki.cern.ch/twiki/bin/view/CMSPublic/LumiPublicResults\#2017_proton_proton_collisions, 2019.

[3] European Organization for Nuclear Research (CERN), High-luminosity large hadron collider (HL-LHC): Technical design report V0.1, CERN-2017-007-M, 2017.

[4] The CMS Collaboration, Technical Design Report: The Phase-2 Upgrade of the CMS Tracker, CERN-LHCC-2017-009, CMS-TDR-014, 2017. 
[5] S.I. Parker, et al., A New architecture for solid state radiation detectors, Nucl. Instrum. Methods Phys. Res. A 395 (1997) 326.

[6] M. Meschini, et. al., First CMS results on 3D pixel sensors interconnected to RD53A readout chip after high energy proton irradiation, Proceedings of the PIXEL2018 conference.

[7] J. Schwandt et. al., CMS Pixel detector development for the HL-LHC, 2018, https://doi.org/10.1016/j.nima.2018.08.121

[8] D. Ruini, et. al., Serial powering for the Phase 2 Upgrade of the CMS Pixel Detector, This conference.

[9] G. Steinbrück et. al., Development of Novel Pixel Sensors for the CMS Upgrade for HL-LHC, Conference Record of the 2018 IEEE NSS/MIC conference, CMS CR-2018/415

[10] J. Christiansen, M. Garcia-Sciveres, et al., $R \& D$ collaboration proposal: Develop- ment of pixel readout integrated circuits for extreme rate and radiation, Technical report CERN-LHCC-2013-008, LHCC-P-006, CERN.

[11] RD53 Collaboration, The RD53A Integrated Circuit, Technical report CERN-RD53-PUB-17-001, CERN, Geneva, 2017.

[12] L. Gaioni et. al, Test results and prospects for RD53A, a large scale $65 \mathrm{~nm}$ CMOS chip for pixel readout at the HL-LHC, 2018, https://doi.org/10.1016/j.nima.2018.11.107

[13] F. Faccio et. al, Radiation-Induced Short Channel (RISCE) and Narrow Channel (RINCE) Effects in 65 and $130 \mathrm{~nm}$ MOSFETs, IEEE Trans. Nuclear Science, vol. 62, pp. 2933, 2015.

[14] M. Menouni, et. al., "1-Grad total dose evaluation of 65nm CMOS technology for the HL-LHC upgrades," J. of Instr., vol. 10, C05009, 2015.

[15] D.B. Ta et. al., Serial powering: Proof of principle demonstration of a scheme for the operation of a large pixel detector at the LHC, Volume 557, pp. 445, 2006.

[16] M. Karagounis et. al, An integrated Shunt-LDO regulator for serial powered systems, In Proceedings of 175 ESSCIRC, pp. 276, 2009.

[17] CMS Collaboration, CMS Technical Design Report for the Pixel Detector Upgrade, CERN-LHCC-2012-016, CMS-TDR-11, 2012. 